\title{
A Review on Graph Based Segmentation
}

\author{
K. Santle Camilus, V. K. Govindan \\ Department of Computer Science and Engineering, National Institute of Technology Calicut \\ Calicut, India. \\ E-mail: camilus@nitc.ac.in
}

\begin{abstract}
Image segmentation plays a crucial role in effective understanding of digital images. Past few decades saw hundreds of research contributions in this field. However, the research on the existence of general purpose segmentation algorithm that suits for variety of applications is still very much active. Among the many approaches in performing image segmentation, graph based approach is gaining popularity primarily due to its ability in reflecting global image properties. This paper critically reviews existing important graph based segmentation methods. The review is done based on the classification of various segmentation algorithms within the framework of graph based approaches. The major four categorizations we have employed for the purpose of review are: graph cut based methods, interactive methods, minimum spanning tree based methods and pyramid based methods. This review not only reveals the pros in each method and category but also explores its limitations. In addition, the review highlights the need for creating a database for benchmarking intensity based algorithms, and the need for further research in graph based segmentation for automated real time applications.
\end{abstract}

Index Terms - Image segmentation, graph based method, boundary detection, graph partitioning algorithm, Image analysis

\section{INTRODUCTION}

Image segmentation can simply result an image partition composed by relevant regions, but the most fundamental challenge in segmentation is to precisely define the spatial extent of some object, which may be represented by the union of multiple regions. In this sense, examples of possible strategies are:

(i) The image is divided into regions and then the object is composed by the union of some of these regions $[1,2]$.

(ii) The approximate location of the object/boundary is found and its spatial extent is defined from that location $[3,4,5,6,7,8]$.

In any case, the strategies are composed by

(a) Object recognition tasks (e.g., regions that compose the object, a point on its boundary, a line inside it, the verification of a segmentation result, the matching between the image and an object model, etc).

(b) Object delineation tasks (e.g., the image partition into regions, pixel classification, optimum boundary tracking, region growing from internal and external seeds, an image-graph cut, etc).

Humans and object models, such as a probabilistic atlas [9] or an active shape model [10], are better for object recognition than computers, but the other way around is true for object delineation. For instance, humans can easily select a point inside the object, but they have difficulties in manual tracing the same boundary several times. In this sense, the most effective approaches are interactive, because they combine the best model for object recognition (the user expert) with the best model for object delineation (some good algorithm). This also makes important the combination of object models for recognition with delineation algorithms for automatic segmentation $[11,12,13]$. Note that, in this scenario, graph-based methods present different graph representations, where the nodes may be pixels $[3,5,14$, $6,7,1,2,8]$, pixel vertices [15, 4], regions [16], or even user-drawn markers [17]. They also differ in the graph algorithm used to solve the problem: graph matching [17], random walker [18], the min-cut/max-flow algorithm [19, 20, 21, 22, 8], Dijkstra's algorithm [3, 15, 4, 23, 14, 6, 7, 2], Kruskal's or Prim's algorithm [24, 25, 26, 27], etc. Note also that, a same graph-search algorithm (e.g., Dijkstra's algorithm) can be used for region-based and boundary-based segmentation, besides other image operators [23]. Therefore, the best way to differ graphbased segmentation methods is by taking into account the graph model and algorithm that they use to solve the segmentation problem. Besides, there are several other approaches in the literature [28, 29, 30], which were not presented as graph-based approaches, but they use similar concepts and can be more efficiently and/or effectively implemented as a graph-based approach $[31,32]$.

Image segmentation produces a set of homogeneous regions of an image such that all pixels of a region are desired to be connected. The integration of all these regions constitutes the entire image. Each region has a set of pixels and each pixel is characterized by its position and feature vector. All pixels of a region are similar with respect to a set of features. The basic principle of most of the graph based segmentation methods is graph partitioning. Each method treats an image as a graph $\mathrm{G}$ in which vertices are composed of pixels. Each edge has a weight generally determined based on the vertices it relates. In graph theory sense, the above segmentation concept is similar to finding a set of sub-graphs $\left\{S G_{1}, S G_{2}, \ldots . . S G_{n}\right\}$ from the graph $\mathrm{G}$ such that for all $k \in\{1,2 \ldots, n\}, \forall i, j$ and $i \neq j, v_{i}, v_{j} \in S G_{k}$ with walks 
between $v_{i}$ and $v_{j}$. The compounding of all the vertices of all the sub-graphs equals the complete set of vertices of the graph. Every sub-graph comprises of a collection of vertices with strong affinities among them. A pictorial representation demonstrating this relation between image segmentation and graph partitioning can be seen in Figure 1.

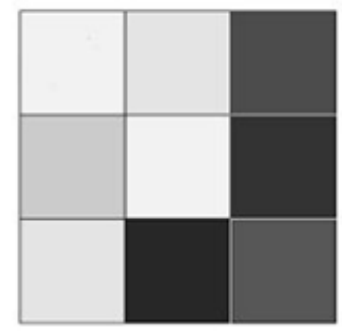

(a)

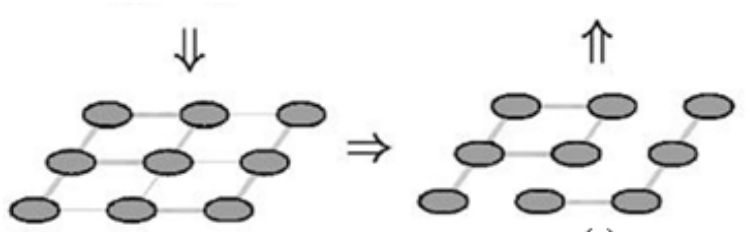

(b)

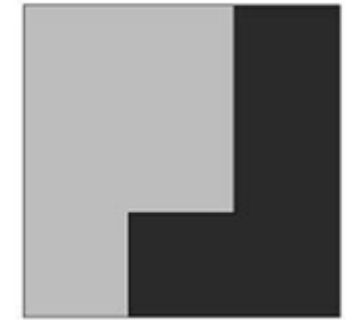

(d)

(c)
Figure 1. Association between image segmentation and graph partitioning.

(a) Image (b) Graph (c) Graph partition (d) Segmented image

The graph partitioning to achieve image segmentation is a challenging problem due to the following questions [33]:

1. What is the precise criterion for a good partition?

2. How can such a partition be computed efficiently?

Due to the subjective nature, defining a good partitioning in segmentation still remains in debate. Assuming this is done, determining a criterion that optimally does this is challenging. Methods that use graphs for image segmentation have been widely investigated within the fields of image processing and image understanding. In these methods, segmentation problems by analogy are translated into graph based problems and that are solved as the graph partitioning problem. These graph based segmentation methods might be grouped as (1) graph cut based methods, (2) interactive methods, (3) minimum spanning tree based methods and (4) pyramid based methods.

The review is organized into eight sections. Section 1, being this introduction describing the graph based approach of segmentation problem. Sections 2 to 5 respectively reviews exhaustively the four classes of methods, namely, graph cut based methods, interactive methods, minimum spanning tree based methods and pyramid based methods. Graph based methods not belonging to the above four categories are briefly reviewed in Section 6. The need for creating a database of images and its ground truth for benchmarking an algorithm in intensity based segmentation research is highlighted in Section 7, and finally the paper is concluded in Section 8 highlighting the major outcome of the review and suggestions for future direction of research.

\section{GRAPH CUT BASED METHODS}

Graph cuts started with the work of Greig [34]. The graph $\mathrm{G}$ can be partitioned into two connected components $\mathrm{A}$ and $\mathrm{B}$ such that $A \cup B=V$ and $A \cap B=\phi$ by omitting the edges linking these two components. The degree of association between A and B can be inferred from the total weight of the discarded edges, which is simply called as a graph cut (a pictorial representation of the graph cut is shown in Figure 2.

$$
\operatorname{cut}(A, B)=\sum_{\substack{u \in A \\ v \in B}} w(u, v)
$$

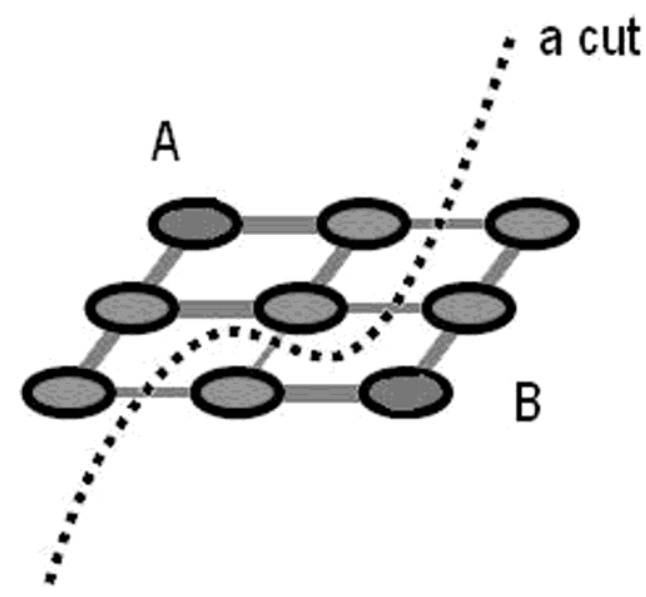

Figure 2. A graph cut

An optimal bi-partition minimizes this graph cut value [33]. By suitably and repeatedly partitioning the graph constructed from an image using the graph cut, different homogeneous regions could be obtained. In another way, each vertex could be considered as a region. By utilising graph cut values, which is a measure to show how much two neighboring regions are homogeneous, regions could be united repeatedly to form image partitions. Major works in this category are the minimum cut algorithm introduced by $\mathrm{Wu}$ and Leahy [35], a normalized cut algorithm by Shi and Malik [33, 36], a variant of normalized cut suggested by Sharon et al. [37], a polynomial time solution to the variant of normalized cut by Hochbaum [38], methods [39, 17, 40] incorporating priors to improve performance of the normalized cut, the average cut by Sarkar et al.[ 41], the min-max cut by Ding et al. [42], and the optimum cut by $\mathrm{Li}$ and Tian [43]. The other important contributions in this category are the minimum mean cut [44] and ratio cut [45] by Wang et al., two methods to determine the global minima of an energy function by Jermyn and Ishikawa [46], an active contours based method by Boykov et al. [47], a heuristic isoperimetric ratio algorithm by Grady and Schwartz [48], a branch-and-bound based technique by Lempitsky et al. [49], Watershed cuts by Cousty et al. [50], ratio regions by Cox et al. [51], min-cut/max-flow algorithms[19-22], 
and a few application focused methods [52-57]. These methods are surveyed briefly in the following.

$\mathrm{Wu}$ and Leahy [35] introduced minimum cut for image segmentation in such a way that the smallest (k-1) cuts among all possible cuts are selected and the corresponding edges are deleted to form k-subgraph partitions. This method favors the formation of very smaller regions, which results in over-segmentation. To overcome this problem, Shi and Malik $[33,36]$ proposed a segmentation method based on normalized cut, the cut cost function that is computed as a fraction of the total edge connections to all the vertices in the graph. The normalized cut, which is a partition criterion of a graph and reflects the global impression of an image, is given as

$$
\operatorname{Ncut}(A, B)=\frac{\operatorname{cut}(A, B)}{\operatorname{assoc}(A, V)}+\frac{\operatorname{cut}(A, B)}{\operatorname{assoc}(B, V)}
$$

Where $\operatorname{assoc}(A, V)=\sum_{u \in A, t \in V} w(u, t) \quad$ is the total link from vertices in A to all vertices in the graph. $\operatorname{assoc}(B, V)$ has similar definition. The authors showed that exact minimization of normalized cut is NPcomplete. Also, they proposed an approximation algorithm for the minimization of the cut cost function by solving a generalized eigenvalue problem. There are four steps in the algorithm: (1) a graph is formed out of an image where vertices are pixels and weight of an edge is a function of similarity between two neighboring pixels. (2) The following eigenvalue system is solved for eigenvectors with the smallest eigenvalues

$$
(D-W) x=\lambda D x
$$

Where $D$ is the diagonal matrix with $d(i)=\sum_{j} w(i, j)$ on its diagonal which is the total connection from vertex $\mathrm{i}$ to all other vertices. $\mathrm{W}$ is the symmetrical matrix with $W(i, j)=w_{i j}$. Also, $\mathrm{x}$ and $\lambda$ are the eignvector and eignvalue respectively. (3) The graph is bi-partitioned using the second smallest eignvector. (4) The above three steps are recursively applied to the two partitions until the normalized cut value is below a threshold. This approximation algorithm is computationally expensive. Also, the normalized cut tends to produce equally sized regions which rarely occur in natural images. The results obtained using this approximation algorithm is presented in Figure 3.

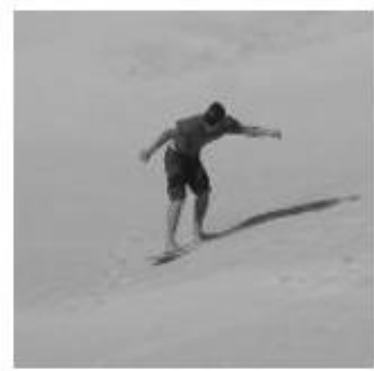

(a)

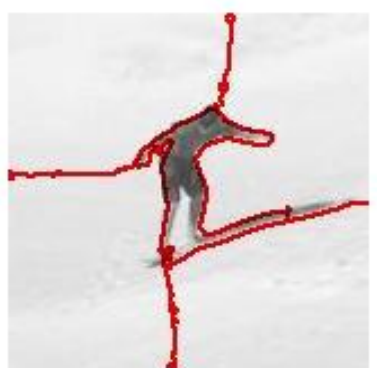

(b)
Figure 3. Results using the normalized cut $[33,36]$. (a) Original image. (b) Segmented image
A variant of normalized cut was discussed by Sharon et al. [37]. It is to minimize the ratio of similarity between the set of regions and its complement and the similarity within the set of regions. Recently, Hochbaum [38] devised a polynomial time solution for this problem which uses a graph cut procedure. A few methods have also been proposed to improve the performances of the normalized cut by incorporating priors. Yu and Shi [39] included a smoothed partial grouping constraint to the normalized cut. Eriksson et al. [17] admitted linear grouping constraints through a Lagrangian dual formation. A constraint normalized cut, which adds prior information in data with explicit linear constraints using an iterative algorithm, was proposed by Xu et al. [40].

The eigenvectors of graph Laplacian or their deviations are normally used to partition a graph in spectral graph partitioning. Sarkar et al. [41] presented a spectral clustering technique based on average cut. This measure is defined as the proposition of the total cut-link weight normalized by the size of the partitions. Ding et al. [42] presented a segmentation technique based on minmax cut criterion. Li et al. [43] suggested optimum cut criterion for partitioning a graph. The optimal solutions to the min-max cut criterion and the optimal cut criterion are all NP-complete [43]. In [44], Wang and Siskind presented a polynomial time algorithm for finding a minimum mean cut. An illustration is given in Figure 4.
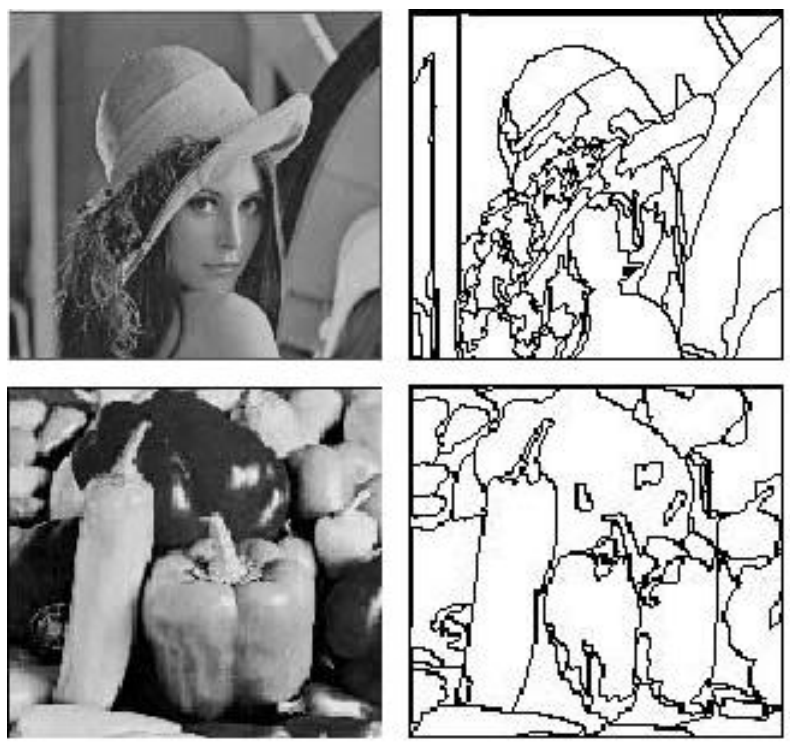

Figure 4. Segmentation of Lena and pepper images using the minimum mean cut [44]

Later on Wang and Siskind [45] examined the minimization of cut ratio cost function and discovered that finding a minimum cut ratio in an arbitrary graph is NP-hard. Hence, they generalized the polynomial time algorithm for finding a minimum mean cut to obtain a polynomial time algorithm for finding a cut that minimizes the cut ratio cost function in connected planar graphs. This solution repeatedly calls an inefficient nonbipartite matching algorithm [38]. Figure 5 shows some results obtained on pepper and medical images. 

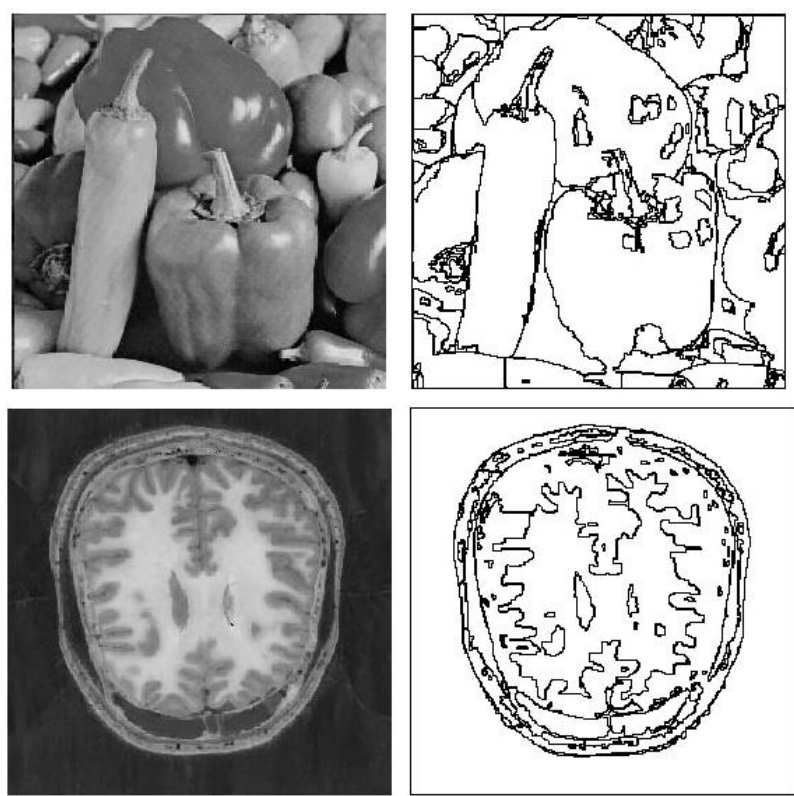

Figure 5. The pepper and medical image segmentation produced by the ratio region method [45]

Jermyn and Ishikawa [46] suggested two methods to determine the global minima of an energy function for the identification of homogeneous regions in images. This energy function is defined on the space of boundaries in the image domain and can incorporate information both from the boundaries and interior of regions. One limitation of this method is that it fails to detect multiply-connected regions. Boykov et al. [47] proposed a method by fusing both active contours and graph cuts. This method demands the extraction of object and background seeds which is hard to obtain for many applications. It can be noted from [58] that determining isoperimetric sets is a NP-hard problem. Grady and Schwartz [48] employed a heuristic algorithm for finding a set with a low isoperimetric ratio in polynomial time, which uses graph cuts, for image segmentation. An instance is given in Figure 6. Lempitsky et al. [49] gave a global optimization framework for image segmentation using the graph cut and branch-and-bound techniques. The worst case running time of this framework is higher. Cousty et al. [50] studied watersheds in edge-weighted graphs under the name watershed cuts. Graph cuts play a significant role in defining these watershed cuts. As the method has a drawback of forming numerous irrelevant smaller regions, it is required to employ a pre-processing andlor a post-processing to obtain the desired outcome. Cox et al. [51] introduced ratio regions for image segmentation. It is to minimize the ratio of the similarity between the set of regions and its complement and the number of regions within the set. A recent study by Hochbaum [38] showed that this problem has a polynomial time solution using graph cuts.
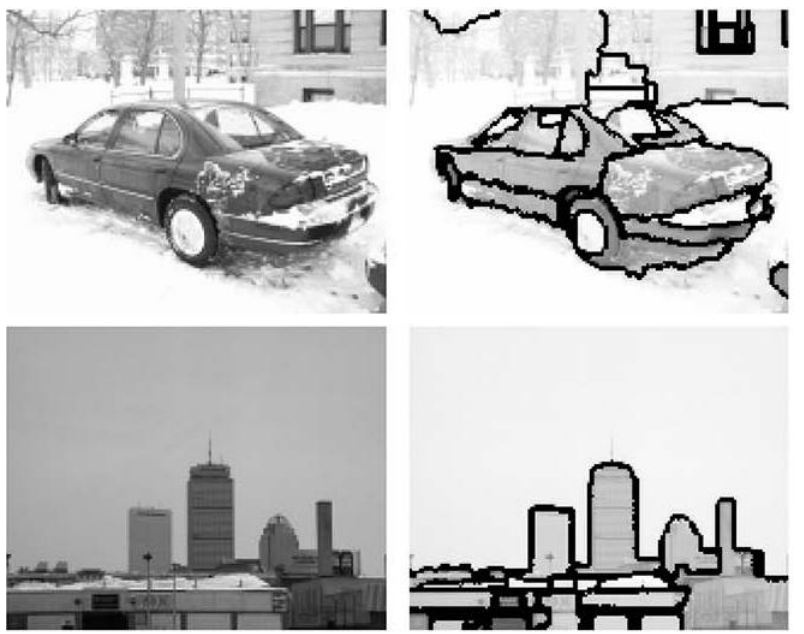

Figure 6. Segmentation results produced by the isoperimetric algorithm [48]

The min-cut/max-flow algorithms, which can be employed for energy functions' minimization, have been used to achieve image segmentation. Kolmogorov et al. [59] provided necessary and sufficient conditions for such energy functions. Geometric properties of regions formed by graph cut methods are detailed in [47]. A few suggested min-cut/max-flow methods for image segmentation in the literature are: push-relabel methods [19], the Dinic algorithm [20], the Boykov method [21] and topological cuts [22]. The push relabel methods [19] maintain a preflow, a flow function. The methods run as long as there is an active vertex in the graph. The push operation increases the flow on a residual edge. A height function on vertices controls which residual edges can be pushed. The height function is altered with relabel operations. The push and relabel operations guarantee that the resulting flow is a maximum flow. A direct application of this method to image segmentation can be seen in the work of Ishikawa and Geiger [60]. The Dinic algorithm [20] pushes flow on non-saturated paths from the source to the sink until the maximum flow in the graph is achieved. The Boykov method [21] works by iteratively repeating the three stages: growing, augmentation and adoption. This method was found to be 2 to 5 times faster than the push-relabel methods and the Dinic algorithm. The drawback of this method is that the augmenting paths found are not necessarily the shortest augmenting paths. Zeng et al. [22] was the first to study about a min-cut/max-flow algorithm that incorporates a topological constraint: the topological cuts problem. It was shown that any optimal solution to the topological cuts problem is NP-hard. Hence, an approximation solution [22] was suggested to solve the topological cuts problem.

Chen et al. [52] used a modified graph cuts based active contours as a fully automatic segmentation method for RNAi fluorescence images. Yang et al. [53] segmented densely packed cells in electron microscopic images via graph cuts. Zhilan et al. [54] employed a graph cut algorithm to segment arbitrary skin regions in images. $\mathrm{Hu}$ et al. [55] extracted clothing by graph cuts. 
This method works based on several assumptions which include cloth arms are similar in color to the torso, which in turn contains dominant colors. These assumptions are not always true as a cloth with sleeves in different colors to the torso is quite common now. Han et al. [56] applied a graph cut based segmentation method to extract white mater, gray matter, and cerebral spinal fluid from brain diffusion tensor image data. Camilus et al. [57] proposed an approach to identify the pectoral muscle in mammograms using a graph cut based merging method and a Bezier curve algorithm. The result of this approach is influenced by the order in which regions are merged in the graph cut based merging method.

An unacceptable running time of a method could prevent its usage in real time applications. Most of the methods in this category are computationally expensive as they are proved to be NP complex and might not be suitable for many real time usages.

\section{INTERACTIVE METHODS}

A large variety of interactive segmentation methods have been developed during the years. In general, none of them is superior to all the others. Also, some methods might be more suited for solving particular segmentation problem better than the others. In short, the main steps of an interactive graph based segmentation method are the following: (1) Get the user preferences and (2) generate an optimal solution (if not, a sub-optimal solution) according to the user preferences and show it. In situations where automatic segmentation is difficult and cannot guarantee correctness or reliability, these interactive methods are best opted. The interactive graph based segmentation methods take the advantage of reliability under users' control. Major research works in this category are live-wire methods [3, 4, 15], a discrete map data structure by Braquelaire et al. [61], an interactive graph cuts method [8,62], methods admitting shape priors into the interactive graph cuts method [6372], methods that improve running time of the interactive graph cuts method [73- 77] and methods which use the interactive graph cuts method in some applications [78, 79]. Other research contributions in this category are the synergistic arch weight estimation approach by Miranda et al. [5], a Grabcut algorithm by Rother et al. [80], a random walker algorithm by Grady [18], an active contours refining method by $\mathrm{Xu}$ et al. [81, 82], and a progressive cut algorithm by Wang et al. [83, 84]. These are briefly reviewed in the following.

In the live-wire method $[3,15]$, the user is required to specify some points in the desired object boundary. The shortest path (using Dijkstra's algorithm), which links all these pre-defined points, is determined. This shortest path makes up the boundary which encloses the desired object. A faster version of live-wire was later introduced by Falcao et al. [4] by devising a linear time graph searching algorithm. Based on segmentation results of 492 medical images, it was observed that this faster version is about 1.3 to 31 times faster than the live wire [4]. An example for the live wire is provided in Figure 7. Braquelaire et al.
[61] presented a discrete map data structure, which uses a region adjacency graph for merging operations, for interactive image segmentation. The discrete map permits a unified representation of both topology and geometry of a segmented image.
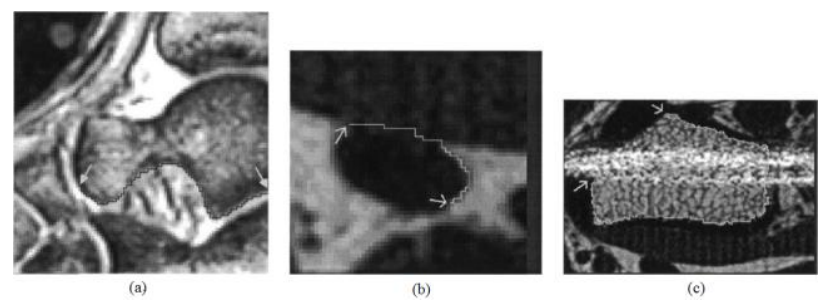

Figure 7. Illustration of the live wire $[4,15]$. The two pre-defined points in the boundary of the desired object are shown using arrows in each case. (a) The detected boundary of the bone talus in an MR image of a foot. (b) A detected vessel in an MR image of a wrist. (c) The detected internal boundary of the cortical part of a bone in the wrist

The interactive graph cuts $[8,62]$ method is a popular method of this category. This method exploits two constraints for segmenting images: hard and soft. Consider $\rho$ contains pixels and $\mathrm{N}$ contains unordered pairs of neighbouring pixels. Let $A=\left(A_{1}, A_{2}, A_{3}, \ldots A_{p}, \ldots \ldots . A_{|\rho|}\right)$ be a segmentation such that $A_{p}$ can either belong to the "background" or "foreground". The soft constraints are defined in the form of a cost function $\mathrm{E}(\mathrm{A})$ that takes into account both boundary and region properties of segments.

$$
E(A)=\lambda \cdot R(A)+B(A)
$$

Where

$$
\begin{gathered}
R(A)=\sum_{p \in \rho} R_{p}\left(A_{p}\right) \\
B(A)=\sum_{\{p, q\} \in N} B_{\{p, q\}} \delta\left(A_{p}, A_{q}\right)
\end{gathered}
$$

And

$$
\delta\left(A_{p}, A_{q}\right)= \begin{cases}1 & \text { if } A_{p} \neq A_{q} \\ 0 & \text { otherwise }\end{cases}
$$

The coefficient $\mathrm{R}_{\mathrm{p}}($.$) and \mathrm{B}_{\{\mathrm{p}, \mathrm{q}\}}$ are region and boundary terms that specifies the penalties for assigning pixel p to "object" and "background", and a penalty for a discontinuity between $\mathrm{p}$ and $\mathrm{q}$ respectively. The coefficient $\lambda$, which specifies the relative importance of the region term against the boundary term, can have values greater than or equal to 0 . The user has to tender hard constraints based on his intension by marking some pixels as foreground/background as

$$
\begin{array}{ll}
\forall p \in O, & A_{p}=\text { 'Object }^{\prime} \\
\forall q \in B, & A_{p}=\text { 'Background }^{\prime}
\end{array}
$$

The idea is to compute the global minimum of the soft constraints among all segmentations A satisfying the hard constraints. This is done using max-flow/min-cut analysis. From an image, a graph with two additional vertices, a "background" terminal and an "object" terminal, is built. The edge set comprises of two types of undirected edges: terminal links (t-links) and neighborhood links (n-links). Each vertex has two t-links connecting to the terminals and the link weights are defined by the regional term and 
hard constraints. Each pair of neighboring pixels is connected by an n-link and its weight is defined by the boundary term. The minimum cost cut of the graph forms the partition between the object and the background. It can be noted that this method also belongs to the classgraph cut based methods. Results of this method are recorded in Figure 8. Apart from its obvious advantage of producing globally optimal solutions, the interactive graph cuts method gives rise to less satisfactory results on images dominated by desired weak contrast boundaries (meaning- these weak contrast boundaries are intended to be identified) and undesired strong contrast boundaries.
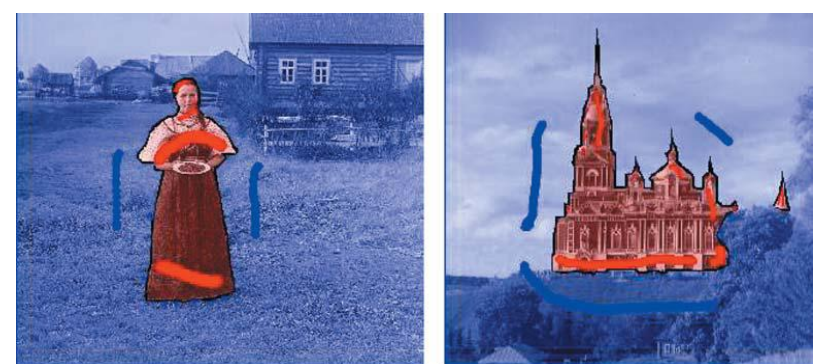

Figure 8. Segmentation of photographs using the interactive graph cuts method [62]. The dark red regions and blue regions are user marked hard constraints for foreground and background respectively, while the corresponding light colors represent segmentation using this method

A few methods admitted shape priors into the interactive graph cuts method to improve its accuracy. Freedman and Zhang [63] embedded shape priors into weights of edges by using a level set formation. Slabaugh and Unal [64] incorporated an elliptical shape prior into the interactive graph cuts method. A user is expected to initialize the segmentation by marking a seed point in an image which evolves later as an ellipse on employing this approach. Zhang et al. [65] also integrated elliptical shape priors into the interactive graph cuts method, but in a different way from the earlier method, to segment cervical lymph nodes on sonograms. Zhu-Jacquot et al. [66] integrated geometric shape priors into the interactive graph cuts method for kidney segmentation from MRI. By making use of the shape information of the heartheart is a compact blob, Funka-Lea et al. [67] included blob constraints to isolate the heart from CT scans. The interactive graph cuts method biases towards shorter boundaries. Das et al. [68], by adding compact shape priors to the interactive graph cuts method, altered its behavior to bias towards larger objects. The interactive graph cuts method is weak in segmenting thin elongated objects. To overcome this problem, Vicente et al. [69] enforced connectivity priors to it. The work also imposes topology [22], specifically, 0-topology. It was shown that the connectivity constraint optimization problems are all NP-hard [69]. Veksler [70] introduced a star shape prior into the graph cuts method. Liu et al. [71] inserted a regional and contour generic shape prior into the graph cuts framework. Kumar et al. [72] formulated OBJ CUT, which uses a loopy belief propagation algorithm to incorporate shape priors into graph cuts. Aside improving the accuracy of the interactive graph cuts method, a few attempts have been made to improve its running time by means of multilevel banded heuristics [73], flow recycling [74], capacity scaling [75], multi-scaling [76], or by planar graph cuts [77]. Weldeselassie and Hamarneh [78] extended the interactive graph cuts to segment diffusion tensor MRI data by making use of tensor calculus and tensor dissimilarity metrics. Malcolm et al. [79] employed the interactive graph cuts method to segment multi-modal tensor valued images by taking into account the Riemannian geometry of the tensor space. Miranda et al. [5] introduced an interactive method for synergistic arch weight estimation, which considers both image attributes and object information. The arch weight estimation finds one of its applications as a basic step in the interactive graph cuts method.

The Grabcut algorithm [80] extracts the foreground of an image, by utilizing a rectangular shaped user's input which roughly holds the foreground, by employing graph cut iteratively. In the random walker algorithm [18], some pixels should be pre-classified by the user. In the subsequent steps, an unclassified pixel is assigned a classified pixel label when a random walker has been given the greatest probability on traversing first to the classified pixel from the unclassified pixel. An example of segmentation results obtained using this random walker algorithm is given in Figure 9.

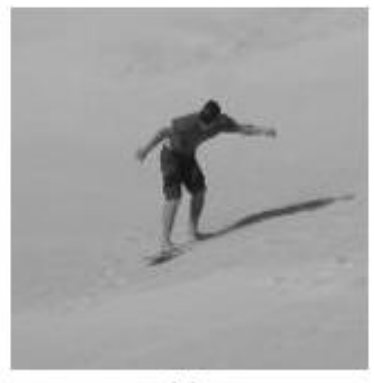

(a)

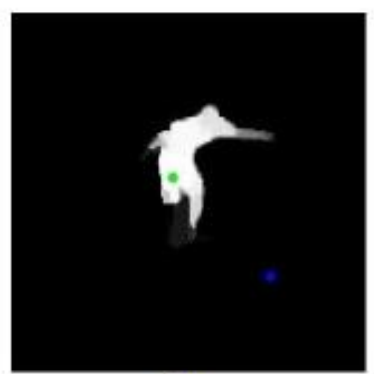

(c)

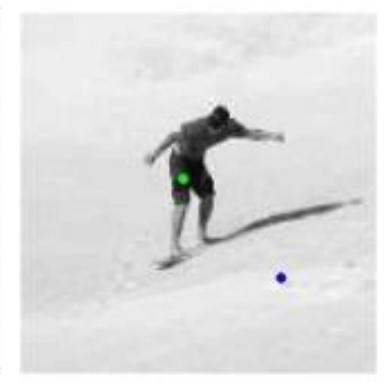

(b)

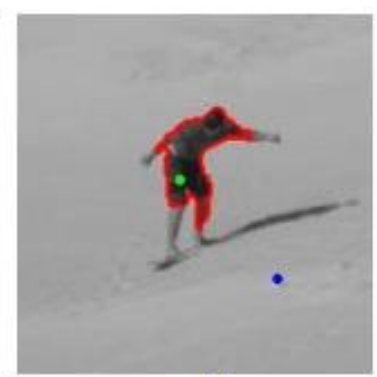

(d)
Figure 9. Results produced by the random walker algorithm [18]. (a) Original image. (b) User defined seeds: foreground- green and background- blue. (c) Probability at each pixel that a random walker released from that pixel reaches the foreground seed. (d) Outlined fore ground (red) by the algorithm

$\mathrm{Xu}$ et al. [81, 82] suggested a segmentation method to refine active contours by iteratively deforming contours using graph cuts. This method also allows an interactive correction of the final boundary if it is not satisfactory. Examples of the segmentation found by the Graph cuts based active contours are displayed in Figure 10. The drawback of this method is that it can segment a single 
object but not multiple objects and also, the graph that is used in this method must be constructed with appropriate pixel connectivity and edge weights. Wang et al. [83, 84] proposed a progressive cut algorithm which explicitly considers user's intention into a graph cut framework for object cutout task.

The interactive graph based methods demand user intervention which is not possible or desirable in many applications. Also, necessary training to the user for interacting to a real time system that uses any of these methods might be required to produce intended results.
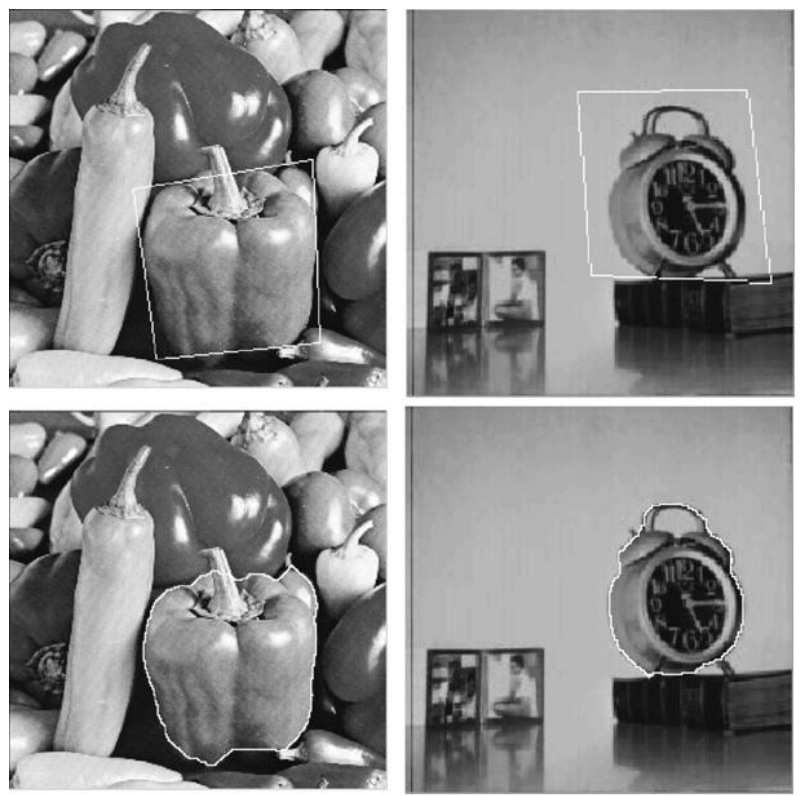

Figure 10. Graph cuts based active contours [82].The two images in the top row show the initialization for graph cuts based active contours. The two images in the bottom row show the corresponding segmentation results using this method

\section{Minimum SPANNING TREE BASED METHODS}

A spanning tree of a connected undirected graph is a subgraph which links all the vertices of the graph and there should be exactly a single path between any two vertices. For the graph, many spanning trees could be found. However, the minimum spanning tree (MST) is a spanning tree whose total weight of edges is less than or equal to the total weight of edges of every other spanning tree. A minimum spanning tree of a graph in which vertices are the pixels and edges represent the similarity between the vertices that it connects, constructed from an image, represents the possible weakest connections. By suitably removing the lowest weighted edges, different partitions that have stronger inherent affinities could be found. Major MST based approaches of segmentation are the method based on Gestalt theory by Zahn [85], a tree partitioning algorithm by Xu et al. [86, 87], an efficient MST algorithm by Felzenszwalb et al. [24, 25] and methods [26, 27] improving the performance of the efficient MST algorithm. A short discussion of these methods is as follow.
Zahn [85] used an approach based on Gestalt theory [88] for detecting and describing clusters. From a given set of points, an MST is constructed first and inconsistent edges in the MST are then deleted to obtain a collection of connected components which in turn constitute clusters. In the method of $\mathrm{Xu}$ et al. [86, 87], a tree partitioning algorithm splits up the MST built from an image into many sub-trees, which represent homogeneous regions, such that each sub-tree should have at least a specific number of vertices and any two nearby sub-trees should feature significantly dissimilar average gray levels. For noisy images, the method yields low quality results due to the incorrect configuration of the MST as an object might be contained in more than one sub-tree due to noise.

Inspired by the work of Zahn, Felzenszwalb et al. [24, 25] presented an efficient graph based method for segmenting images using MST and is widely used as it runs in video rate in practice. The method works with the assumption that edges between vertices in the same segment should have relatively low weights than edges between vertices in different segments. Initially each vertex is considered as a segment. Then in a greedy way, two segments are repeatedly selected to consider for merging. Based on a comparison predicate, the decision of merging the two segments is made. The comparison predicate is defined based on the internal difference of a segment and difference between two segments. The internal difference of a segment(S) is the largest weight in the minimum spanning tree of the segment, which is given by

$$
\operatorname{int}(s)=\max _{e=\operatorname{MST}(S, E)}\left(w_{e}\right)
$$

The difference between two segments is calculated as the minimum weight edge connecting the two segments. When the difference between two segments is less than or equal to the minimum of any of the internal difference of the two segments, then the predicate allows the two segments to be merged. The authors showed that the segmentation produced by this method is neither too coarse nor too fine. Also, any attempt to change the definition of the difference between two segments (say, median edge weight instead of minimum weight edge between the two segments) to make the method more robust lead to the solution NP-hard. As the method merges two segments based on a single low weight edge between them, there are possibilities that the results could considerably be affected by noise if no initial filtering of the image is done. A sample result obtained using this method is shown in Figure 11. Fahad et al. [26] and Zhang et al. [27] suggested some modifications to improve its performance.

In practical scenarios, acquiring images without noise is almost impossible due to the perplexed imaging environment. Usage of this category of methods in those noisy images without pre-processing that includes filtering might yield unacceptable segmentation as the MST based methods are very much susceptible to noise. 


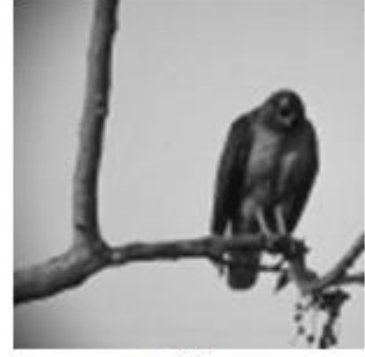

(a)

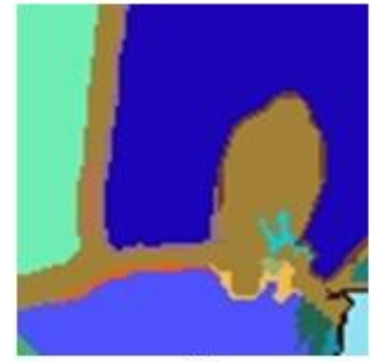

(b)
$\mathrm{F}$

igure 11. Results obtained for the efficient graph based method [24, 25]. (a) Original image. An initial Gaussian filtering of the original image was performed before segmentation by setting sigma $=0.5$. (b) Segmented image

\section{PYRAMID BASED METHODS}

A review of pyramidal structures that are used for image segmentation can be found in [16]. The general framework involves the creation of a graph from the original image. From this base graph, a set of graphs defined in multi-level of resolution, which can be visualized as a pyramid, is built. The vertices and edges at level $\mathrm{L}+1$ are formed from the reduction of vertices and edges at level L using a reduction function. A level of pyramid called as working level is chosen as the responsible level to yield segmentation. Based on the working principle to build pyramids, they can be classified into two categories: (1) regular pyramids and (2) irregular pyramids

\section{A. Regular pyramids}

In regular pyramids, spatial relationships and the reduction factor, which is defined as the ratio between the number of vertices at level $\mathrm{L}$ and the number of vertices at level $\mathrm{L}+1$, are constant and fixed, hence, the size and the layout of the structure of the pyramids are predicable. The first segmentation method based on regular pyramids was proposed by Chen and Pavlidis [89]. Ping et al. [90] utilized a pyramid built using a Gaussian filter function having adjustable filter scales. Burt et al. [91] proposed a pyramid linking approach to attain image segmentation. But the accuracy of this method is sensitive to the right selection of the working level. To overcome this drawback, a modified pyramid linking approach which particularly uses two scaling rules was suggested by Ziliani and Jensen [92]. Though this method attempted to improve the pyramid linkage approach, a few drawbacks remain in common to both these approaches. They are: elongated regions are not properly segmented, and the structure of the pyramid varies even due to small rotations, shifts and scales of the input image [93].

\section{B. Irregular pyramids}

In opposite to the regular ones, spatial relationships and the reduction factors are not constants in irregular pyramids; hence, the size and the layout are not predictable. However, these types of pyramid solve the problems associated with the regular pyramids such as shift variance and inability to segment elongated objects. A few segmentation methods have been proposed based on irregular pyramids. Montanvert et al. [94] exploited a hierarchy of region adjacency graph, which performs stochastic decimation which in turn uses two binary state variables and a random variable, to achieve segmentation This method yields different segmentation results depending on different outcomes of the random variable for the same input settings. To overcome this drawback, Jolion and Montanvert [95] proposed an adaptive pyramid in which the random variable is replaced by an interest variable in the decimation process. A localized pyramid was proposed by Huart et al. [96] to achieve segmentation. The bounded irregular pyramid was proposed by Marfil et al. [97] which combines features from regular and irregular pyramids. Brun and Kropatsch [98] combined a combinatorial pyramid and a union-findbased decimation algorithm to attain segmentation.

With the aid of empirical methods, Marfil et al. [16] showed that irregular pyramids yield better results than regular pyramids in segmenting objects. Though irregular pyramids resolve the problems posed by the regular pyramids, they possess unpredictable structures which account for unbounded execution time for local operations within each level.

\section{OTHER METHODS}

There are many other methods which do not belong to any of the above categories of the graph based methods. Methods based on graph-searching principles [99-102] are notable of this kind. A few methods were proposed for image segmentation based on graph shortest path algorithms [103, 104, 23]. It was shown that shortest paths, random walker, and watershed were all actually the same algorithm with different norms [105]. The work of Gomez et al. [106] utilized an iterative binary coloring technique, which considers the changing behavior of adjacent pixels, for image segmentation. Granularity of the output of this method mainly depends on number of iterations: a few iterations might develop a wrong segmentation, while too much iteration might yield useless results. Corso et al. [107] proposed an extended graph-shift algorithm, which is a hierarchical energy minimization algorithm, for segmenting brain tumors and multiple sclerosis lesions in MRI. Yuan et al. [108] proposed a multi-modal segmentation method based on region fusion and narrow band energy graph partitioning. Ta et al. [109] utilized a framework of graph-based tools for microscopic image segmentation.

\section{ISSUES IN PERFORMANCE EVALUATION}

Each graph based segmentation technique addresses a solution to some specific segmentation problems. Due to this, a technique might work well for a particular set of images but fail to segment satisfactorily for another set of images. Authors tested their algorithms using limited private set of images or by using standard test images like Lena, photographer, baboon and so on and compare the 
performance with other algorithms using qualitative evaluation orland one or more quantitative criteria [110112]. The main concern is whether the chosen dataset represent all necessary test scenarios like different illuminations, variations, conditions (say, day and night) and contrasts. Given this, creating a database of images and its ground truth for benchmarking an algorithm in intensity based segmentation would be an interesting area of research for computer vision and image processing community for two primary reasons: (1) to evaluate an algorithm with different test images which represent all possible test scenarios and (2) to compare different algorithms in a common platform and rank them based on their performance. Without this dataset and ground truth, it is very difficult to evaluate and compare different graph based segmentation methods. Though at this moment a few benchmarking systems are publically available, they are not very much suitable for evaluating an algorithm in intensity based segmentation. For example, most of the test cases of the Berkeley segmentation dataset and benchmarking [113] estimate the ability of an algorithm in identifying textures.

\section{CONCLUSION}

Recently, there has been increasing interest in using graph based methods as a powerful tool for segmenting images. This review has discussed some of the major graph based methods and highlighted their strengths as well as limitations. Some difficulties of these methods have brought down their use in practical applications. The primary reason is the higher computational complexity. The search for a vertex or an edge in a graph requires polynomial time. At the same time, search for a match of a scene model to an object model in the graph has exponential complexity [114]. The current research in graph based methods orients towards producing approximate solution (sub-optimal solution) for such graph matching problem to reduce processing time. Also, use of a priori information that include shape, topology and appearance model of the category of images to be segmented is getting more popularity. Apart from the class-wise disadvantages in general, in fact, many of the graph based segmentation methods have their own disadvantages. This survey demonstrates that most of the methods fail to find use in automated real time applications which normally work with no human involvement and demand acceptable running time with robust behavior of the methods. This demonstrates the need for further research to refine the graph based segmentation techniques to be applied for automatic real time scenarios. A few important future direction of research can be: (1) incorporation of the fuzzy set theory into graph based frameworks to achieve enhanced segmentation performances. (2) Use of multi-criteria to partition a graph to achieve an efficient segmentation solution is a promising direction of research. (3) Constructing a graph using feature sets rather than pixel level information and finding an optimum partition that maximizes the dissimilarities across boundaries is also a study of interest to the research community.

\section{REFERENCES}

[1] Noma, A., Graciano, A., Consularo, L., Cesar, M. R. $\&$ Bloch, I. (2008). A new algorithm for interactive structural image segmentation. arXiv:0805.1854v2 [cs.CV].

[2] Rocha, L.M., Cappabianco, F.A.M., \& Falcao, A.X. (2009). Data clustering as an optimum-path forest problem with applications in image analysis. International Journal of Imaging Systems and Technology, 19(2), 50-68.

[3] Mortensen, E. (1998). Interactive segmentation with intelligent scissors. Graphical Models and Image Processing, 60 (5), 349-384.

[4] Falcao, A. X., Udupa, J. K., \& Miyazawa, F. K. (2000). An ultra-fast user-steered image segmentation paradigm: live wire on the fly. IEEE Transaction on Medical Imaging, 19 (1), 55-62.

[5] De Miranda, P. A. V., Falcão, A. X., \& Udupa, J. K. (2010). Synergistic arc-weight estimation for interactive image segmentation using graphs. Computer Vision and Image Understanding, 114 (1), 85-99.

[6] Bai, X., \& Sapiro G. (2007). Distance cut: interactive segmentation and matting of images and videos. IEEE International Conference on Image Processing, San Antonio, Texas, 2, pp. 249-252.

[7] Protiere, A., \& Sapiro, G. (2007). Interactive image segmentation via adaptive weighted distances. IEEE Transactions on Image Processing, 16(4), 1046-1057.

[8] Boykov, Y. Y., \& Jolly M. P. (2001). Interactive graph cuts for optimal boundary \& region segmentation of objects in n-d images. Eighth IEEE International Conference on Computer Vision, 1, pp. 105-112.

[9] Talairach, J., \& Tournoux P. (1988). Co-planar stereotaxic atlas of the human brain. New York: Thieme Medical Inc.

[10] Cootes, T., Taylor, C., Cooper, D., \& Graham, J. (1995). Active shape models-their training and application. Computer Vision and Image Understanding, 61(1), 38-59.

[11] Grau, V., Mewes, A.U.J., Alcaniz, M., Kikinis, R., \& Warfield, S.K. (2004). Improved watershed transform for medical image segmentation using prior information. IEEE Transactions on Medical Imaging, 23(4), 447-458.

[12] Liu, J., \& Udupa, J.K. 2009. Oriented active shape models. IEEE Transactions on Medical Imaging, 28(4), 571-584.

[13] Miranda, P.A.V., Falcao, A.X., \& Udupa, J.K. (2009). Cloud bank: a multiple clouds model and its use in MR brain image segmentation. IEEE International Symposium on Biomedical Imaging, Boston, MA, pp. 506-509. 
[14] Miranda, P.A.V., \& Falcao, A.X. (2009). Links between image segmentation based on optimumpath forest and minimum cut in graph. Journal of Mathematical Imaging and Vision, 35(2), 128-142.

[15] Falcao, A. X., Udupa, J. K., Samarasekera, S., Sharma, S., Hirsch, B. E., \& Lotufo R. de. A. (1998). User-steered image segmentation paradigms: live wire and live lane. Graphical Models and Image Processing, 60 (4), 233-260.

[16] Marfil, R., Molina-Tanco, L., Bandera, A., Rodriguez, J. A., \& Sandoval F. (2006). Pyramid segmentation algorithms revisited. Pattern Recognition, 39 (8), 1430-1451.

[17] Eriksson, A. P., Olsson, C., \& Kahl, F. (2007). Normalized cuts revisited: a reformulation for segmentation with linear grouping constraints. International Conference on Computer Vision, pp. $1-8$.

[18] Grady, L. (2006). Random walks for image segmentation. IEEE Transactions on Pattern Analysis and Machine Intelligence, 28 (11), 17681783.

[19] Goldberg, A. V., \& Tarjan R. E. (1988). A new approach to the maximum-flow problem. Journal of the ACM, 35 (4), 921-940.

[20] Dinic, E. A. (1980). Algorithm for solution of a problem of maximum flow in networks with power estimation. Soviet Mathematics- Doklady, 11, 1277-1280.

[21] Boykov, Y., \& Kolmogorov, V. (2004). An experimental comparison of $\mathrm{min}-\mathrm{cut} / \mathrm{max}$ - flow algorithms for energy minimization in vision. IEEE Transactions on Pattern Analysis and Machine Intelligence, 26 (9), 1124-1137.

[22] Zeng, Y., Samaras, D., Chen W., \& Peng, Q. (2008). Topology cuts: a novel min-cut/max-flow algorithm for topology preserving segmentation in $\mathrm{n}-\mathrm{d}$ images. Computer Vision and Image Understanding, 112 (1), pp. 81-90.

[23] Falcao, A. X., Stolfi, J., \& Alencar, De. (2004). The image foresting transform: theory, algorithms, and applications. IEEE Transactions on Pattern Analysis and Machine Intelligence, 26 (1), pp. 1929.

[24] Felzenszwalb, P., \& Huttenlocher, D. (2004). Efficient graph-based image segmentation. International Journal of Computer Vision, 59 (2), 167-181.

[25] Felzenszwalb, P.F., \& Huttenlocher, D.P. (1998). Image segmentation using local variation. IEEE Computer Society Conference on Computer Vision and Pattern Recognition, pp. 98-104.

[26] Fahad, A., \& Morris, T. (2006). A faster graphbased segmentation algorithm with statistical region merge. Lecture Notes in Computer Science, pp. 286-293.

[27] Zhang, M., \& Alhajj, R. (2006). Improving the graph-based image segmentation method. Eighteenth IEEE International Conference on Tools with Artificial Intelligence, pp. 617 - 624.
[28] Saha, P.K., \& Udupa, J.K. (2001). Relative fuzzy connectedness among multiple objects: theory, algorithms, and applications in image segmentation. Computer Vision and Image Understanding, 82 (1), 42-56.

[29] Ciesielski, K.C., Udupa, J.K., Saha, P.K., \& Zhuge Y. (2007). Iterative relative fuzzy connectedness for multiple objects with multiple seeds. Computer Vision and Image Understanding, 107(3), 160-182.

[30] Vincent, L., \& Soille, P. (1991). Watersheds in digital spaces: an efficient algorithm based on immersion simulations. IEEE Transaction on Pattern Analysis and Machine Intelligence, 13(6), 583-598.

[31] Ciesielski, K.C., Udupa, J.K., Falcao, A.X., \& Miranda, P.A.V. (2011). Fuzzy connectedness and graph cut image segmentation - similarities and differences. Proceedings of SPIE on Medical Imaging, To appear.

[32] Audigier, R., \& Lotufo, R.A. (2007). Watershed by image foresting transform, tie-zone, and theoretical relationship with other watershed definitions. Eighth International Symposium on Mathematical Morphology and its Applications to Signal and Image Processing, Rio de Janeiro, Brazil, pp. $277-$ 288.

[33] Shi, J., \& Malik, J. (2000). Normalized cuts and image segmentation. IEEE Transactions on Pattern Analysis and Machine Intelligence, 22 (8), 888-905.

[34] Greig, D.M., Porteous, B.T., \& Seheult A.H. (1989). Exact maximum a posteriori estimation for binary images. Journal of the Royal Statistical Society Series B, 51, 271-279.

[35] Wu, Z., \& Leahy, R. (2002). An optimal graph theoretic approach to data clustering: theory and its application to image segmentation. IEEE Transactions on Pattern Analysis and Machine Intelligence, 15 (11), 1101-1113.

[36] Shi, J., \& Malik, J. (1997). Normalized cuts and image segmentation. IEEE Computer Society Conference on Computer Vision and Pattern Recognition, pp. 731-737.

[37] Sharon, E., Galun, M., Sharon, D., Basri, R., \& Brandt, A. (2006). Hierarchy and adaptivity in segmenting visual scenes. Nature, 442 (7104), 810813.

[38] Hochbaum, D. S. (2010). Polynomial time algorithms for ratio regions and a variant of normalized cut. IEEE Transactions on Pattern Analysis and Machine Intelligence, 32 (5), 889-898.

[39] Yu, S. X., \& Shi, J. (2004). Segmentation given partial grouping constraints. IEEE Transactions on Pattern Analysis and Machine Intelligence, 26 (2), 173-183.

[40] Xu, L., Li, W., \& Schuurmans, D. (2009). Fast normalized cut with linear constraints. IEEE Conference on Computer Vision and Pattern Recognition, pp. 2866-2873.

[41] Sarkar, S., \& Soundararajan, P. (2000). Supervised learning of large perceptual organization: graph 
spectral partitioning and learning automata. IEEE Transactions on Pattern Analysis and Machine Intelligence, 22 (5), 504-525.

[42] Ding, C. H. Q., He, X., Zha, H., Gu, M., \& Simon, H. D. (2001). A min-max cut algorithm for graph partitioning and data clustering. International Conference on Data Mining, pp. 107-114.

[43] Li, X., \& Tian, Z. (2007). Optimum cut-based clustering. Signal Processing, 87(11), 2491-2502.

[44] Wang, S., \& Siskind, J. M. (2001). Image segmentation with minimum mean cut. Eighth IEEE International Conference on Computer Vision, 1, pp. 517-524.

[45] Wang, S., \& Siskind, J. M. (2003). Image segmentation with ratio cut. IEEE Transactions on Pattern Analysis and Machine Intelligence, 25 (6), 675-690.

[46] Jermyn, I., \& Ishikawa, H. (2001). Globally optimal regions and boundaries as minimum ratio weight cycles. IEEE Transactions on Pattern Analysis and Machine Intelligence, 23 (10), 1075-1088.

[47] Boykov, Y., \& Kolmogorov, V. (2003). Computing geodesics and minimal surfaces via graph cuts. Ninth IEEE International Conference on Computer Vision, 1, pp. 26-33.

[48] Grady, L., \& Schwartz, E. L. (2006). Isoperimetric graph partitioning for image segmentation. IEEE Transactions on Pattern Analysis and Machine Intelligence, 28 (3), 469-475.

[49] Lempitsky, V., Blake, A., \& Rother, C. (2008) . Image segmentation by branch-and-mincut. European Conference on Computer Vision, pp. 1529.

[50] Cousty, J., Bertrand, G., Najman, L., and Couprie, M. (2009). Watershed cuts: minimum spanning forests and the drop of water principle. IEEE Transactions on Pattern Analysis and Machine Intelligence, 31 (8), 1362-1374.

[51] Cox, I. J., Rao, S. B., \& Zhong, Y. (1996). Ratio regions: a technique for image segmentation. International Conference on Pattern Recognition, 2, pp. 557-564.

[52] Chen, C., Li, H., \& Zhou, X. (2007). Automated segmentation of drosophila RNAi fluorescence cellular images using graph cuts. Lecture Notes in Computer Science, pp. 116-125.

[53] Yang, H., \& Choe, Y. (2009). Cell tracking and segmentation in electron microscopy images using graph cuts, Sixth IEEE International Conference on Symposium on Biomedical Imaging, pp. 306309.

[54] Zhilan, H., Guijin, W., Xinggang, L., \& Hong, Y. (2009). Skin segmentation based on graph cuts. Tsinghua Science and Technology, 14 (4), 478-486.

[55] Hu, Z., Yan, H., \& Lin, X. (2008). Clothing segmentation using foreground and background estimation based on the constrained delaunay triangulation. Pattern Recognition, 41 (5), 15811592.
[56] Han, D., Singh, V., Lee, J. E., Zakszewski, E., Adluru, N., Oakes, T. R., \& Alexander, A. (2009). An experimental evaluation of diffusion tensor image segmentation using graph-cuts. Conference of the IEEE Engineering in Medicine and Biology Society, 5653-5656.

[57] Camilus, K. S., Govindan, V. K., \& Sathidevi, P. S. (2010). Computer aided identification of the pectoral muscle in digitized mammograms. Journal of Digital Imaging, 23 (5), 562-580.

[58] Mohar, B. (1989). Isoperimetric numbers of graphs. Journal of Combinatorial Theory Series B, 47 (3), 274-291.

[59] Kolmogorov, V., \& Zabin, R. (2004). What energy functions can be minimized via graph cuts? IEEE Transactions on Pattern Analysis and Machine Intelligence, 26 (2), 147-159.

[60] Ishikawa, H., \& Geiger, D. (1998). Segmentation by grouping junctions. IEEE Computer Society Conference on Computer Vision and Pattern Recognition, pp. 125-131.

[61] Braquelaire, J. P., \& Brun, L. (1998). Image segmentation with topological maps and inter-pixel representation. Journal of Visual Communication and Image Representation, 9(1), 62-79.

[62] Boykov, Y., \& Funka-Lea, G. (2006). Graph cuts and efficient $n-d$ image segmentation. International Journal of Computer Vision, 70 (2), 109-131.

[63] Freedman, D., \& Zhang, T. (2005). Interactive graph cut based segmentation with shape priors, IEEE Computer Society Conference on Computer Vision and Pattern Recognition, 1, pp.755-762.

[64] Slabaugh, G., \& Unal, G. (2005). Graph cuts segmentation using an elliptical shape prior, IEEE International Conference on Image Processing, 2, pp. 1222-1225.

[65] Zhang, J., Wang, Y., \& Shi, X. (2009). An improved graph cut segmentation method for cervical lymph nodes on sonograms and its relationship with node's shape assessment. Computerized Medical Imaging and Graphics, 33, 602-607.

[66] Zhu-Jacquot, J., \& Zabih, R. (2007). Graph cuts segmentation with statistical shape priors for medical images, Third International IEEE Conference on Signal-Image Technologies and Internet-Based System, pp.631-635.

[67] Funka-Lea, G., Boykov, Y., Florin, C., Jolly, M. P., Moreau-gobard, R., Ramaraj, R., \& Rinck, D. (2006). Automatic heart isolation for CT coronary visualization using graph-cuts. IEEE International Symposium on Biomedical Imaging, pp. 614-617.

[68] Das, P., \& Veksler, O. (2006). Semiautomatic segmentation with compact shape prior. 3rd Canadian Conference on Computer and Robot Vision, pp. 28-36.

[69] Vicente, S., Kolmogorov, V., \& Rother, C. (2008). Graph cut based image segmentation with connectivity priors. IEEE Conference on Computer Vision and Pattern Recognition, pp.1-8. 
[70] Veksler, O. (2008). Star shape prior for graph-cut image segmentation, 10th European Conference on Computer Vision, pp. 454-467.

[71] Liu, C., Li, F., Zhang, Y., \& Gu, H. (2009). Interactive image segmentation based on hierarchical graph-cut optimization with generic shape prior. Lecture Notes in Computer Science, pp. 201-210.

[72] Kumar, M. P., Torr, P. H. S., \& Zisserman, A. (2005). OBJ CUT. IEEE Computer Society Conference on Computer Vision and Pattern Recognition, 1, pp. 18-25.

[73] Lombaert, H., Sun, Y., Grady, L., and Xu, C. (2005). A multilevel banded graph cuts method for fast image segmentation, Tenth IEEE International Conference on Computer Vision, 1, pp. 259-265.

[74] Kohli, P., \& Torr, P. H. S. (2007). Dynamic graph cuts for efficient inference in Markov random fields. IEEE Transactions on Pattern Analysis and Machine Intelligence, 29 (12), 2079-2088.

[75] Juan, O., \& Boykov,Y. (2007). Capacity scaling for graph cuts in vision. IEEE 11th International Conference on Computer Vision, pp.1-8.

[76] Delong, A., \& Boykov, Y. (2008). A scalable graph-cut algorithm for N-D grids. IEEE Conference on Computer Vision and Pattern Recognition, pp.1-8.

[77] Schmidt, F. R., Toppe, E., \& Cremers, D. (2009). Efficient planar graph cuts with applications in computer vision. IEEE Conference on Computer Vision and Pattern Recognition, pp. 351-356.

[78] Weldeselassie, Y. T., \& Hamarneh, G. (2007). DTMRI segmentation using graph cuts. Proceedings of SPIE, pp. 1-9.

[79] Malcolm, J., Rathi, Y., and Tannenbaum, A. 2007. A graph cut approach to image segmentation in tensor space, Proceedings of Workshop Component Analysis Methods, pp. 18-25.

[80] Rother, C., Kolmogorov, V., \& Blake, A. (2004). Grabcut: interactive foreground extraction using iterated graph cuts. ACM Transactions on Graphics, 23, 309-314.

[81] Xu, N., Bansal, R., \& Ahuja, N. (2003). Object segmentation using graph cuts based active contours. Computer Vision and Pattern Recognition Conference, 2, 46-53.

[82] Xu, N., Ahuja, N., \& Bansal, R. (2007). Object segmentation using graph cuts based active contours. Computer Vision and Image Understanding, 107 (3), 210-224.

[83] Yang, Q., Tang, X., Wang, C., Ye, Z., \& Chen, M. (2007). Progressive cut: an image cutout algorithm that models user intentions. IEEE Multimedia, 14 (3), 56-66.

[84] Wang, C., Yang, Q., Chen, M., Tang, X., \& Ye, Z. (2006). Progressive cut, Proceedings of the 14th Annual ACM international Conference on Multimedia, pp. 251-260.

[85] Zahn, C. T. (1971). Graph-theoretical methods for detecting and describing gestalt clusters. IEEE Transactions on Computers, C-20 (1), 68-86.
[86] Xu, Y., Olman, V., \& Uberbacher, E. C. (1996). A segmentation algorithm for noisy images. IEEE International Joint Symposia on Intelligence and Systems, pp. 220-226.

[87] Xu, Y., \& Uberbacher, E. C. (1997). 2D image segmentation using minimum spanning trees. Image and Vision Computing, 15 (1), pp. 47-57.

[88] Wertheimer, M. (1938). Laws of organization in perceptual forms- A source book of Gestalt psychology. London: Routledge \& Kegan Paul.

[89] Chen, P.C., \& Pavlidis, T. (1980). Image segmentation as an estimation problem, Computer Graphics and Image Processing, 12 (2), 153-172.

[90] Ping, Y., Runsheng, W., \& Diannong, L. (1996). A new image segmentation approach based on linked pyramid. Proceedings of International Conference on Signal Processing, pp. 1118-1121.

[91] Burt, P., Hong, T., \& Rosenfeld, A. (1981). Segmentation and estimation of image region properties through cooperative hierarchical computation. IEEE Transaction on Systems, Man and Cybernetics, 11 (12), 802-809.

[92] Ziliani, F., \& Jensen, B. (1998). Unsupervised image segmentation using the modified pyramidal linking approach. International Conference on Image Processing, 3, pp. 303-307.

[93] Bister, M., Cornelis, J., Rosenfeld, A. (1990). A critical view of pyramid segmentation algorithms. Pattern Recognition Letters, 11(9), 605- 617.

[94] Montanvert, A., Meer, P., \& Rosenfeld, A. (1991). Hierarchical image analysis using irregular tessellations, IEEE Transaction on Pattern Analysis Machine Intelligence, 13(4), 307-316.

[95] Jolion, J. M., \& Montanvert, A. (1992). The adaptive pyramid, a framework for 2D image analysis, Computer Vision, Graphics, and Image Processing, 55(3), 339-348.

[96] Huart, J., \& Bertolino, P. (2005). Similarity-based and perception-based image segmentation. IEEE International Conference on Image Processing, pp. 1148-1151.

[97] Marfil, R., Rodriguez, J. A., Bandera, A., \& Sandoval, F. (2004). Bounded irregular pyramid: a new structure for colour image segmentation. Pattern Recognition, 37 (3), 623-626.

[98] Brun, L., \& Kropatsch, W. G. (2003). Construction of combinatorial pyramids. Lecture Notes in Computer Science, pp. 1-12.

[99] Montanari, U. (1971). On the optimal detection of curves in noisy pictures, Communications of ACM, 14(5), pp. 335-345.

[100]Martelli, A. (1972). Edge detection using heuristic search methods. Computer Graphics and Image Processing, 1(2), pp. 169-182.

[101]Martelli, A. (1976). An application of heuristic search methods to edge and contour detection, Communications of the ACM, 19(2), 73-83.

[102]Pope, D. L., Parker, D. L., Clayton, P. D., \& Gustafson, D. E. (1985). Left ventricular border 
recognition using a dynamic search algorithm. Radiology, 155 (2), 513-518.

[103]Sonka, M., Winniford, M. D., \& Collins, S. M. (1995). Robust simultaneous detection of coronary borders in complex images. IEEE Transactions on Medical Imaging, 14 (1), pp. 151-161.

[104]Udupa, J. K., Saha, P. K., \& Lotufo, R. A. (2002). Relative fuzzy connectedness and object definition: theory, algorithms, and applications in image segmentation. IEEE Transactions on Pattern Analysis Machine Intelligence, 24 (11), 1485-1500.

[105]Couprie, C., Grady, L., Najman, L., \& Talbot, H. (2009). Power watersheds: a new image segmentation framework extending graph cuts, random walker and optimal spanning forest. International Conference on Computer Vision, pp. 731-738.

[106]Gomez, D., Montero, J., Yáñez, J., \& Poidomani, C. (2007). A graph coloring approach for image segmentation. Omega, 35 (2), 173-183.

[107]Corso, J. J., Yuille, A., Sicotte, N. L., \& Toga, A. (2007). Detection and segmentation of pathological structures by the extended graph-shifts algorithm, International Conference on Medical Image Computing and Computer-Assisted Intervention, pp. 985-993.

[108]Yuan, X., Situ, N., \& Zouridakis, G. A. (2009). Narrow band graph partitioning method for skin lesion segmentation. Pattern Recognition, 42 (6), 1017-1028.

[109]Ta, V., Lézoray, O., Elmoataz, A., \& Schüpp, S. (2009). Graph-based tools for microscopic cellular image segmentation. Pattern Recognition, 42 (6), 1113-1125.

[110]Zhang, Y. (1996) . A survey on evaluation methods for image segmentation. Pattern Recognition, 29 (8), 1335-1346.

[111]Unnikrishnan, R., Pantofaru, C., \& Hebert, M. (2007). Toward objective evaluation of image segmentation algorithms. IEEE Transactions on Pattern Analysis and Machine Intelligence, 29 (6), 929-944.

[112]Zhang, H., Fritts, J., \& Goldman, S. (2008). Image segmentation evaluation: A survey of unsupervised methods, Computer Vision and Image Understanding, 110 (2), 260-280.

[113]Martin, D., Fowlkes, C., Tal, D., \& Malik, J. (2001) A database of human segmented natural images and its application to evaluating segmentation algorithms and measuring ecological statistics. International Conference on Computer Vision, 2, pp 416-423.

[114]Sanfeliu, A., Alquezar, R., Andrade, J., Climent, J., Serratosa, F. \& Verges, J. (2002). Graph-based representations and techniques for image processing and image analysis. Pattern Recognition, 35 (3), 639-650.

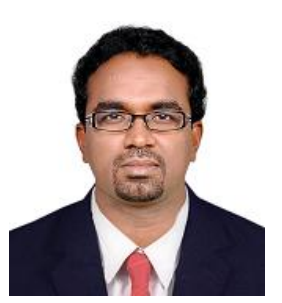

Dr. K. Santle Camilus is currently working as Technical lead at Samsung India Software Operations, Bangalore, India. He received his Bachelor's degree from Madras University in information technology in the year 2003 and Master's degrees in Computer Science and Engineering from Manonmaniam Sundaranar University in the year 2005. He received his $\mathrm{PhD}$ degree in medical image analysis from National Institute of Technology, Calicut, India in the year 2011. He has over 2 years of industrial experience and 1 year of teaching experience. His research areas of interest include image processing, pattern recognition and medical image processing. He has over 12 research publications in various international journals and conferences. He has reviewed papers for many conferences and journals including Springer and Elsevier. His biography appeared in the 29th edition of Who's Who in the World.

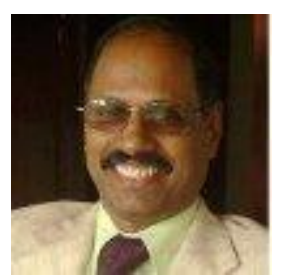

Dr. V. K. Govindan is currently serving as Professor of Computer Science and Engineering Department and Dean Academic, National Institute of Technology, Calicut, India. $\mathrm{He}$ received Bachelor's and Master's degrees in Electrical Engineering from National Institute of Technology (the erstwhile Regional Engineering College), Calicut in the year 1975 and 1978, respectively. He was awarded $\mathrm{PhD}$ in Character Recognition from the Indian Institute of Science, Bangalore, in 1989. He has over 32 years of teaching experience in the capacity of Lecturer (1979-87), Asst. professor (1987-98) and Professor (1998 onwards). He was Head of the Department of Computer Science and Engineering during January 2000 to August 2005. His research areas of interest include medical imaging, agent technology, biometrics based authentication, data compression, and distributed computing. He has over 85 research publications in various international journals and conferences, and authored several books on Operating systems and Computer basics. He has reviewed papers for many conferences and journals including IEEE Transactions and evaluated several $\mathrm{PhD}$ theses. 